\title{
OPEN Determination of the pathogenicity of known COL4A5 intronic variants by in vitro splicing assay
}

Received: 25 April 2019

Accepted: 15 August 2019

Published online: 03 September 2019
Tomoko Horinouchi $\mathbb{D}^{1}$, Kandai Nozu ${ }^{1}$, Tomohiko Yamamura ${ }^{1}$, Shogo Minamikawa ${ }^{1}$, China Nagano ${ }^{1}$, Nana Sakakibara ${ }^{1}$, Koichi Nakanishi ${ }^{2}$, Yuko Shima $\mathbb{D}^{3}{ }^{3}$, Naoya Morisada ${ }^{1}$, Shinya Ishiko ${ }^{1}$, Yuya Aoto ${ }^{1}$, Hiroaki Nagase ${ }^{1}$, Hiroki Takeda ${ }^{1}$, Rini Rossanti ${ }^{1}$, Hiroshi Kaito $\mathbb{D}^{1}$, Masafumi Matsuo ${ }^{4}$ \& Kazumoto lijima ${ }^{1}$

X-linked Alport syndrome (XLAS) is a congenital renal disease caused by mutations in COL4A5. In XLAS cases suspected of being caused by aberrant splicing, transcript analysis needs to be conducted to determine splicing patterns and assess the pathogenicity. However, such analysis is not always available. We conducted a functional splicing assay using a hybrid minigene for seven COL4A5 intronic mutations: one was identified by us and six were found in the Human Gene Mutation Database. The minigene assay revealed exon skipping in four variants, exon skipping and a 10-bp insertion in one variant, and no change in one variant, which appeared not to be pathogenic. For one variant, our assay did not work. The results of all three cases for which transcript data were available were consistent with our assay results. Our findings may help to increase the accuracy of genetic test results and clarify the mechanisms causing aberrant splicing.

COL4A5 (NM: 000495.4) is the causative gene of X-linked Alport syndrome (XLAS). XLAS can cause end-stage renal disease accompanied by sensorineural hearing loss and ocular abnormalities in affected patients ${ }^{1}$. It has been reported that $13.7 \%$ or $14.9 \%$ of pathogenic COL $4 A 5$ mutations are splice site mutations ${ }^{2,3}$. However, in these previous studies, no intronic variants leading to aberrant COL4A5 splicing outside of the consensus sequence (AG-GT) were identified. Many unclassified pathogenic intronic or exonic mutations result in splicing abnormalities $^{4-6}$. We previously reported that, of 41 families with COL4A5 aberrant splicing, only 32 (78\%) had splice site variants, while the others had deep intronic or exonic variants ${ }^{5}$. Clinically diagnosed XLAS cases lacking COL4A5 exonic or consensus splice site variants can be caused by intronic or synonymous exonic splicing abnormalities.

Recently, we conducted a genetic analysis of inherited kidney diseases. However, it is sometimes difficult to distinguish intronic variants leading to splicing errors from harmless polymorphisms. The most reliable method to identify splicing aberrations is by in vivo assay. This method is based on the analysis of mRNA derived from the affected tissue. However, this sample type is not always available. Instead, we analyse mRNA isolated from peripheral blood leukocytes, which is unstable and easily destroyed during transportation. To prevent RNA destruction, we use the Paxgene Blood RNA Kit (Qiagen Inc., Chatsworth, CA) or RNAlater RNA Stabilization Reagent (Qiagen Inc.). However, even using these tools, it is sometimes difficult to extract mRNA of sufficient quantity and quality.

Several in silico approaches have been developed to assess the influence of sequence variants on splicing ${ }^{7}$, but the information acquired using these approaches remains incomplete ${ }^{8,9}$. They can provide information about changes in the ability of splicing-related proteins to bind to each splicing related element, but do not accurately predict splicing patterns. Recently, the in vitro splicing assay with hybrid minigene has emerged as a relatively fast approach to identify splicing aberrations in inherited kidney disease ${ }^{10-13}$. Three reports have shown that the hybrid minigene system, using HEK293 or HEK293T cells derived from human embolic kidney cells, was

${ }^{1}$ Department of Pediatrics, Kobe University Graduate School of Medicine, 7-5-1 Kusunoki-cho, Chuo, Kobe, Hyogo, 650-0017, Japan. ${ }^{2}$ Department of Child Health and Welfare (Pediatrics), Graduate School of Medicine, University of the Ryukyus, 207, Uehara, Nishihara-cho, Tyutou, Okinawa, 903-0125, Japan. ${ }^{3}$ Department of Pediatrics, Wakayama Medical University, 811-1, Kimiidera, Wakayama, Wakayama Prefecture, 641-8510, Japan. ${ }^{4}$ Department of Physical Therapy, Faculty of Rehabilitation, Kobe Gakuin University, 518, Arise, Ikawadani-cho, Nishi, Kobe, Hyogo, 651-2180, Japan. Correspondence and requests for materials should be addressed to K.Nozu (email: nozu@med.kobe-u.ac.jp) 


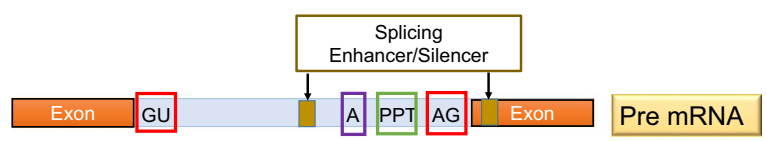

Figure 1. Schema for splicing regulation. Exons, intron, splice consensus sequences (GU or AG), U2 branch point (A), polypyrimidine tract (PPT), splicing enhancer/silencer.

effective for assessing COL4A5 variant splicing patterns ${ }^{5,14,15}$. Currently, the best approach to identify splicing mutations with a role in disease is to use a combination of these in vivo, in silico and in vitro analyses ${ }^{7}$.

Many of the components contributing to accurate intron splicing are already known. These include the splicing regulator, consensus sequence, branch point, exonic/intronic splicing enhancer/silencer sequence, and polypyrimidine tract (Fig. 1$)^{16}$. Therefore, we focused on previously reported COL4A5 intronic mutations separated from exons. We obtained the sequences of all reported disease-causing COL4A5 mutations (at intronic positions 10 to $40 \mathrm{bp}$ upstream of the subsequent exon) from The Human Gene Mutation Database (HGMD) and added one from our case ${ }^{3,17-21}$. Using a splicing reporter minigene system, we introduced the different mutations and investigated splicing pattern changes. Finally, we speculate on the mechanisms of the splicing pattern changes using evidence from in silico analyses.

\section{Results}

The minigene assay revealed aberrant splicing in five of seven variants. We observed exon skipping in four variants (No. 1, No. 3, No. 5 and No. 7), both exon skipping and a 10-bp insertion in one variant (No. 2), and no change in one variant (No. 6) (Table 1, Fig. 2 and Supplementary Fig. 2). Analysis of minigene construct No. 4 (intron 28-14T $>$ A), containing introns 28-29, was not possible. The inserted sequence produced numerous nonspecific bands, making it difficult to assess the splicing pattern. Among three of the seven variants (No. 2, No. 5 and No. 7), our in vitro assay results were identical to the previously reported patient transcripts (Table 1). In these cases, mRNA samples were extracted from skin fibroblasts (No. 2), hair roots (No. 5) or peripheral blood leukocytes (No. 7). The results of in silico analysis are shown in Table 1. A marked decrease in the original acceptor site score was found for No. 2, No. 4 and No. 5. This suggests that the corresponding changes made it more difficult for the original acceptor site to function in exonic recognition. Novel splicing acceptor sites were created in No. 2, No. 4 and No. 7, suggesting the possibility of insertion or deletion. Indeed, No. 2 leads to a 10-bp insertion in addition to exon 9 skipping (Supplementary Fig. 3). In three variants, reduced polypyrimidine tract scores were observed (No. 3, No. 5 and No. 6). The polypyrimidine tract is preceded by a branch point sequence and is important for downstream exonic recognition. A reduced polypyrimidine tract score may lead to impaired exonic recognition. However, minigene analysis showed no change in splicing pattern in No. 6, even though a reduced polypyrimidine tract score was observed. Of the six cases in which in vitro results were obtained, in four cases (No. 1, No. 2, No. 3 and No. 5) the results could be predicted to some extent by the in silico results, while in No. 6 and No. 7 prediction from the in silico results was difficult (Table 1). At present, this in silico analysis is insufficient to predict the precise splicing pattern. In all cases, no branch point alteration was observed.

\section{Discussion}

This is the first report comprehensively exploring COL4A5 splicing patterns in variants assumed to be pathogenic in intronic regions 10 to 40 bp upstream of exons. Although XLAS is a monogenic disease caused by COL4A5, pathogenic COL4A5 mutations are not detected in all clinically diagnosed XLAS patients. In such cases, intronic mutations or exonic synonymous mutations can cause aberrant splicing ${ }^{4-6}$. Moreover, $15-60 \%$ of pathogenic mutations cause genetic disease through pre-mRNA splicing abnormalities ${ }^{22}$. Therefore, a renewed focus should be placed on intronic variants that can cause splicing abnormalities.

Recently, splicing has been focused on as a target of treatment. Modifying the splicing pattern is an important goal of some molecular therapies ${ }^{23}$. Oligonucleotide-based therapies such as Nusinersen or Eteplirsen have been approved by the $\mathrm{FDA}^{24,25}$. Clarification of the involvement of splicing in disease pathogenicity can lead to the development of treatments. For XLAS, we recently reported the phenotype-genotype correlation restricted to cases with COL4A5 splicing abnormalities. These results suggested the possibility of oligonucleotide therapy leading to exon skipping in patients with XLAS ${ }^{5}$. Taken together, these results show that determining the involvement of splicing in the pathogenicity of inherited kidney diseases is important and may inform treatment approaches.

When nucleotide changes are detected in a gene, it is important to determine whether or not they are pathogenic. This is often difficult, particularly when variants are found in introns. To assess the pathogenicity of intronic variants, it is important to conduct transcript analysis. Tools for in silico analysis, such as the Human Splicing Finder (http://www.umd.be/HSF3/), can be used to predict the effect of transcriptional variants and may aid in predicting the disruption of the original consensus splice sites. We previously reported that, among $41 \mathrm{fam}$ ilies with COL4A5 variants inducing aberrant splicing, 19 were completely compatible with the Human Splicing Finder results. Of the remaining families, 17 yielded data that enabled prediction of splicing defects, a precise novel splice site could not be predicted, and the remaining five families yielded insufficient data for the prediction of splicing abnormalities ${ }^{5}$. In this study, No. 6 showed decreasing polypyrimidine tract scores, but in vitro results showed no difference between this patient and the control. Therefore, the limitation of in silico analysis lies in accurately predicting splicing patterns.

The most accurate method to predict pathogenicity is to conduct transcriptional analysis using the patients' affected organ tissues. However, obtaining kidney specimens to observe tissue-specific gene expression is always 


\begin{tabular}{|c|c|c|c|c|c|c|c|c|c|}
\hline & \multirow[b]{2}{*}{ gDNA mutation } & \multirow[b]{2}{*}{ mRNA } & \multicolumn{3}{|c|}{ In vitro (minigene) } & \multicolumn{3}{|l|}{ In silico } & \multirow[b]{2}{*}{ Reference } \\
\hline & & & $\begin{array}{l}\text { Genetic region } \\
\text { cloned }\end{array}$ & Cloning via & Result & $\begin{array}{l}\text { HSF (original } \\
\text { ASS) }\end{array}$ & $\begin{array}{l}\text { HSF } \\
\text { (novel ASS) }\end{array}$ & $\begin{array}{l}\text { SVM-BPF } \\
\text { (PPT score) }\end{array}$ & \\
\hline No. 1 & IVS8-17 $\mathrm{T}>\mathrm{G}$ & N/A & Intron8-10 & Restriction and ligation & Ex9 skipping & $7.54 \rightarrow 6.44$ & & $\rightarrow$ & Nagel et al. ${ }^{17}$ \\
\hline No. 2 & IVS8-12 G > A & $\begin{array}{l}\text { Ex9 skipping/ } \\
\text { 10 bp ins }\end{array}$ & Intron8-10 & Restriction and ligation & $\begin{array}{l}\text { Ex9 skipping/ } \\
10 \text { bp ins }\end{array}$ & $7.54 \rightarrow 2.74$ & $1 \rightarrow 3.4$ & $\rightarrow$ & Wang et al. ${ }^{18}$ \\
\hline No. 3 & IVS21-20 T $>A$ & N/A & Intron21-22 & Restriction and ligation & Ex22 skipping & $8.09 \rightarrow 5.99$ & & $\downarrow$ & $\begin{array}{l}\text { Bekheirnia } \\
\text { et al. }^{3}\end{array}$ \\
\hline No. 4 & IVS28-14 T >A & N/A & Intron28-29 & In-fusion reaction & N/A & $3.87 \rightarrow 1$ & $1 \rightarrow 4.68$ & $\rightarrow$ & Weber et al. ${ }^{19}$ \\
\hline No. 5 & IVS31-10 T >G & Ex 32 skipping & Intron30-33 & In-fusion reaction & Ex32 skipping & $5.68 \rightarrow 1$ & & $\downarrow$ & King et al. ${ }^{20}$ \\
\hline No. 6 & IVS37-11 C>A & N/A & Intron37-39 & In-fusion reaction & No change & $6.45 \rightarrow 4.92$ & & $\downarrow$ & Martin et al. ${ }^{21}$ \\
\hline No. 7 & IVS26-18 A>G & Ex27 skipping & Intron26-28 & Restriction and ligation & Ex27 skipping & $12.03 \rightarrow 10.7$ & $1 \rightarrow 6.97$ & $\rightarrow$ & Our case \\
\hline
\end{tabular}

Table 1. mRNA, in vitro (minigene) and in silico assays. IVS: intron, N/A: not available, Ex: exon. HSF: Human Splicing Finder, ASS: acceptor site score, SVM-BPF: SVM-BPfinder, PPT: polypyrimidine tract.
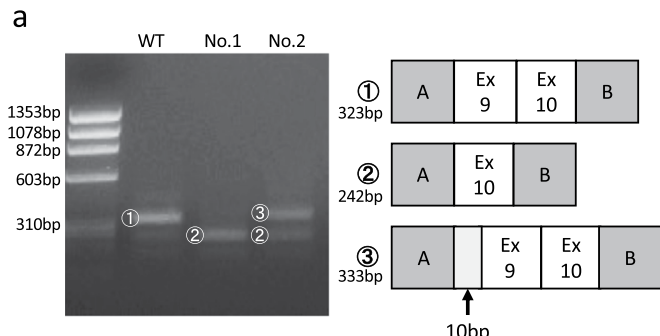

b

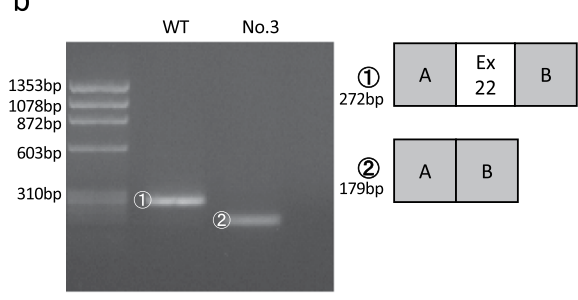

C

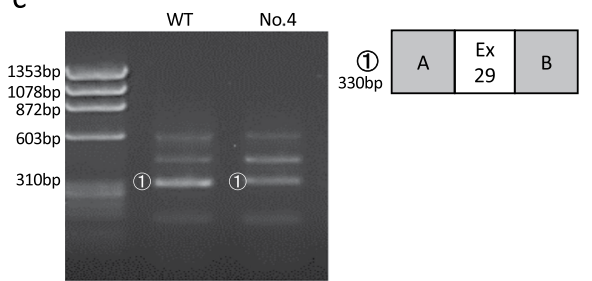

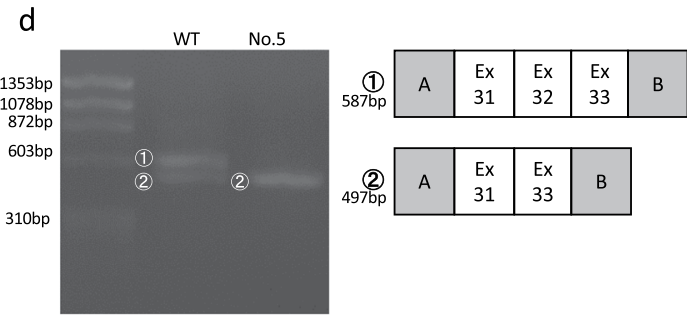

e
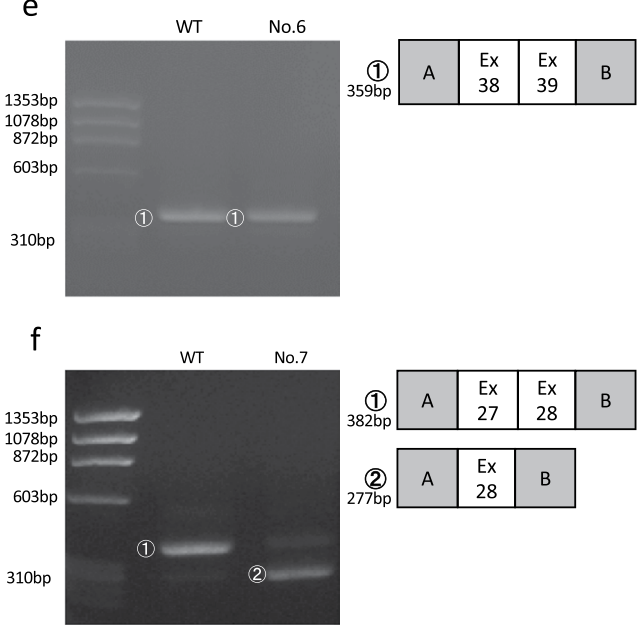

Figure 2. Minigene assay transcript analysis. Electrophoresis results and schematic transcript analysis from the minigene constructs. The direct sequence is shown in Supplementary Fig. 2. (a) Wild type (WT) exhibited a single band (full) and No. 1 (case) exhibited a single band (exon 9 skipping), while No. 2 (case) exhibited double bands (exon 9 skipping and 10-bp insertion). (b) WT exhibited a single band (full) and No. 3 (case) exhibited a single band (exon 22 skipping). (c) Both WT and No. 4 (case) exhibited many bands, which were difficult to compare. (d) WT exhibited double bands (full and exon 32 skipping) and No. 5 (case) exhibited a single band (exon 32 skipping). (e) Both WT and No. 6 (case) exhibited single bands (full). (f) WT exhibited a single band (full) and No. 7 (case) exhibited a single band (exon 27 skipping). No cropped pictures are used in this figure.

difficult. In addition to renal tissue, peripheral blood lymphocytes, skin biopsies, hair roots and urine-derived cells from patients have also been reported as adjuncts for the diagnosis of $\mathrm{AS}^{5,6,14,18,20,26-29}$. It may be possible to determine the pathogenicity of a mutation by examining the mRNA extracted from each sample. However, this approach is suboptimal as transcripts are not stable within tissues, and abnormal transcripts can be quickly degraded by nonsense-mediated mRNA decay ${ }^{30}$. Instead, it has been reported that it is effective to examine the expression of type IV collagen $\alpha 5$ chain in the hair root, which can be collected noninvasively, but the sensitivity and specificity have not been fully established ${ }^{14}$. Therefore, there remains a serious need for another system to assess the pathogenicity of variants of uncertain significance.

Recently, a splicing assay with a hybrid minigene approach has been established as a relatively fast and accurate means to identify splicing aberrations and to study their underlying functional mechanisms ${ }^{10-15,31}$. This 
approach has been validated by reports of the same results being obtained from in vivo and in vitro studies ${ }^{10-12,32}$. In our study, in three of the seven patient transcript analyses, the obtained results were completely consistent with our in vitro assay results. In one case, our minigene system did not work, possibly because of the low original acceptor site score. In addition, the results of No. 5 minigene showed normal and skipping bands in the WT, but only skipping bands in a patient (Fig. 2d). The possibility of identifying abnormal splicing bands in the WT has already been reported in other COL $4 A 5$ studies with minigene assay ${ }^{5,15}$, which can lead to false positive results of aberrant splicing. Therefore, the in vitro results should be evaluated in comparison with controls, bearing in mind variation in splicing patterns among different tissues and differences between in vitro and in vivo conditions.

In silico analysis assisted the interpretation of in vivo or in vitro analysis results, but was insufficient to predict the splicing pattern by itself. Three intronic variants analyzed by COL $4 A 5$ minigene assay have been reported so far ${ }^{5,14,15}$. Among them, the coexistence of exon skipping and a 43-bp deletion was detected in one case. It was possible to predict exon skipping by in silico analysis from reduced branch point scores in that case; however, the 43-bp deletion could not be predicted. Furthermore, although exon skipping occurred in the remaining two cases, their splice site scores decreased only slightly, supporting the difficulty of predicting exon skipping by in silico analysis itself (Supplementary Table 1). However, although it is difficult to predict the splicing pattern, clarifying the mechanisms of aberrant splicing by in silico analysis allows us to understand how to correct the splicing pattern to weaken or ameliorate the pathogenicity. Combining in vivo and/or in vitro and in silico analyses can thus be a powerful tool for assessing pathogenicity and for the development of appropriate therapeutic approaches.

In conclusion, our splicing assay with hybrid minigene makes it possible to assess whether the mutation in question causes aberrant splicing. In addition, in silico tools can predict the aberrant splicing mechanisms. Our system is useful to increase the accuracy of genetic tests through determining the pathogenicity of intronic mutations and may help inform treatment discovery strategies.

\section{Methods}

In vitro splicing assay. To create hybrid minigene constructs, we used the $\mathrm{H} 492$ vector that we developed previously, which is based on the pcDNA 3.0 mammalian expression vector and contains a multicloning site (Invitrogen, Carlsbad, CA, USA) (Supplementary Fig. 1) ${ }^{10}$. We cloned DNA fragments containing a couple of exons and introns around the target variant in the COL4A5 gene using classical restriction and ligation methods or In-fusion cloning methods, as shown in Table 1. As for No. 1-3 and No. 7, cloning was performed by classical restriction and ligation methods using NheI and BamHI. As for No. 4-6, we used infusion cloning methods with the HD Cloning Kit (Takara Bio Inc., Tokyo, Japan), in accordance with the manufacturer's instructions. As for No. 7, the patients' gDNA was available, so gDNA of the patients and WT was cloned. As for No. 1-6, because the patients' gDNA was not available (just the sequence was reported), we initiated cloning with WT gDNA and then introduced mutations by site-directed mutagenesis using PrimeSTAR mutagenesis basal kit (Takara Bio Inc.), in accordance with the manufacturer's instructions. The primers used are shown in Supplementary Table 1.

The hybrid minigenes were confirmed by sequencing and transfected into HEK293T and HeLa cells using Lipofectamine ${ }^{\circledR} 2000$ (Thermo Fisher Scientific, Waltham, MA, CA). Total RNA was extracted from cells after $24 \mathrm{~h}$ using the Rneasy ${ }^{\circledR}$ Plus Mini Kit (QIAGEN, GmbH, Hilden, Germany). Total RNA ( $\left.1 \mu \mathrm{g}\right)$ was reverse-transcribed using RNA to cDNA EcoDry Premix (Double Primed) (Takara Bio Inc). PCR was performed using a forward primer corresponding to a segment upstream of exon A (YH307: 5'-ATTACTCGCTCAGAAGCTGTGTTGC-3') and a reverse primer complementary to a segment downstream of exon B (YH308: 5'-CTGCCAGTTGCTAAGTGAG AGACTT-3'). PCR products were analysed by electrophoresis on an $1.5 \%$ agarose gel using $\varphi$ X174-Hae III digest marker and direct sequencing.

In silico splicing assay. We predicted splicing domain strength in each variant, using Human Splicing Finder (http://www.umd.be/HSF3/) and SVM-BP Finder (http://regulatorygenomics.upf.edu/Software/SVM_ $\mathrm{BP} /)^{33,34}$. As for potential splice sites, scores obtained using MaxEnt Scan matrix are shown.

Compliance with ethical standards. All procedures were reviewed and approved by the Institutional Review Board of Kobe University School of Medicine.

\section{Data Availability}

The datasets generated and/or analysed during the current study are available from the corresponding author on reasonable request.

\section{References}

1. Kashtan, C. E. Alport syndrome and thin glomerular basement membrane disease. Journal of the American Society of Nephrology: JASN 9, 1736-1750 (1998).

2. Jais, J. P. et al. X-linked Alport syndrome: natural history in 195 families and genotype- phenotype correlations in males. Journal of the American Society of Nephrology: JASN 11, 649-657 (2000).

3. Bekheirnia, M. R. et al. Genotype-phenotype correlation in X-linked Alport syndrome. Journal of the American Society of Nephrology: JASN 21, 876-883, https://doi.org/10.1681/asn.2009070784 (2010).

4. Fu, X. J. et al. X-linked Alport syndrome associated with a synonymous p.Gly292Gly mutation alters the splicing donor site of the type IV collagen alpha chain 5 gene. Clinical and experimental nephrology 20, 699-702, https://doi.org/10.1007/s10157-015-1197-9 (2016).

5. Horinouchi, T. et al. Detection of Splicing Abnormalities and Genotype-Phenotype Correlation in X-linked Alport Syndrome. Journal of the American Society of Nephrology: JASN 29, 2244-2254, https://doi.org/10.1681/asn.2018030228 (2018).

6. Nozu, K. et al. X-linked Alport syndrome caused by splicing mutations in COL4A5. Clinical journal of the American Society of Nephrology: CJASN 9, 1958-1964, https://doi.org/10.2215/cjn.04140414 (2014).

7. Baralle, D., Lucassen, A. \& Buratti, E. Missed threads. The impact of pre-mRNA splicing defects on clinical practice. EMBO reports 10, 810-816, https://doi.org/10.1038/embor.2009.170 (2009). 
8. Hartmann, L., Theiss, S., Niederacher, D. \& Schaal, H. Diagnostics of pathogenic splicing mutations: does bioinformatics cover all bases? Frontiers in bioscience: a journal and virtual library 13,3252-3272 (2008).

9. Houdayer, C. et al. Evaluation of in silico splice tools for decision-making in molecular diagnosis. Human mutation 29, 975-982, https://doi.org/10.1002/humu.20765 (2008).

10. Nozu, K. et al. In vivo and in vitro splicing assay of SLC12A1 in an antenatal salt-losing tubulopathy patient with an intronic mutation. Human genetics 126, 533-538, https://doi.org/10.1007/s00439-009-0697-7 (2009).

11. Nakanishi, K. et al. A comparison of splicing assays to detect an intronic variant of the OCRL gene in Lowe syndrome. European journal of medical genetics, https://doi.org/10.1016/j.ejmg.2017.08.001 (2017).

12. Yamamura, T. et al. An in vitro splicing assay reveals the pathogenicity of a novel intronic variant in ATP6V0A4 for autosomal recessive distal renal tubular acidosis. BMC nephrology 18, 353, https://doi.org/10.1186/s12882-017-0774-4 (2017).

13. Yamamura, T. et al. Functional splicing analysis in an infantile case of atypical hemolytic uremic syndrome caused by digenic mutations in C3 and MCP genes. Journal of human genetics 63, 755-759, https://doi.org/10.1038/s10038-018-0436-9 (2018).

14. Malone, A. F., Funk, S. D., Alhamad, T. \& Miner, J. H. Functional assessment of a novel COL4A5 splice region variant and immunostaining of plucked hair follicles as an alternative method of diagnosis in X-linked Alport syndrome. Pediatric nephrology (Berlin, Germany) 32, 997-1003, https://doi.org/10.1007/s00467-016-3565-4 (2017).

15. Chiereghin, C. et al. Alport syndrome cold cases: Missing mutations identified by exome sequencing and functional analysis. PloS one 12, e0178630, https://doi.org/10.1371/journal.pone.0178630 (2017).

16. Buratti, E., Baralle, M. \& Baralle, F. E. Defective splicing, disease and therapy: searching for master checkpoints in exon definition. Nucleic acids research 34, 3494-3510, https://doi.org/10.1093/nar/gk1498 (2006).

17. Nagel, M., Nagorka, S. \& Gross, O. Novel COL4A5, COL4A4, and COL4A3 mutations in Alport syndrome. Human mutation 26, 60, https://doi.org/10.1002/humu.9349 (2005).

18. Wang, F., Wang, Y., Ding, J. \& Yang, J. Detection of mutations in the COL4A5 gene by analyzing cDNA of skin fibroblasts. Kidney international 67, 1268-1274, https://doi.org/10.1111/j.1523-1755.2005.00204.x (2005).

19. Weber, S. et al. Identification of 47 novel mutations in patients with Alport syndrome and thin basement membrane nephropathy. Pediatric nephrology (Berlin, Germany) 31, 941-955, https://doi.org/10.1007/s00467-015-3302-4 (2016).

20. King, K., Flinter, F. A., Nihalani, V. \& Green, P. M. Unusual deep intronic mutations in the COL4A5 gene cause X linked Alport syndrome. Human genetics 111, 548-554, https://doi.org/10.1007/s00439-002-0830-3 (2002).

21. Martin, P. et al. Spectrum of COL4A5 mutations in Finnish Alport syndrome patients. Human mutation 15, 579, https://doi. org/10.1002/1098-1004(200006)15:6<579::Aid-humu13>3.0.Co;2-k (2000).

22. Wang, G. S. \& Cooper, T. A. Splicing in disease: disruption of the splicing code and the decoding machinery. Nature reviews. Genetics 8, 749-761, https://doi.org/10.1038/nrg2164 (2007).

23. Sazani, P. \& Kole, R. Therapeutic potential of antisense oligonucleotides as modulators of alternative splicing. The Journal of clinical investigation 112, 481-486, https://doi.org/10.1172/jci19547 (2003).

24. Traynor, K. Eteplirsen approved for Duchenne muscular dystrophy. American journal of health-system pharmacy: AJHP: official journal of the American Society of Health-System Pharmacists 73, 1719, https://doi.org/10.2146/news160063 (2016).

25. Ottesen, E. W. ISS-N1 makes the First FDA-approved Drug for Spinal Muscular Atrophy. Translational neuroscience 8, 1-6, https:// doi.org/10.1515/tnsci-2017-0001 (2017)

26. Hashimura, Y. et al. Milder clinical aspects of X-linked Alport syndrome in men positive for the collagen IV alpha5 chain. Kidney international 85, 1208-1213, https://doi.org/10.1038/ki.2013.479 (2014).

27. Nakanishi, K. et al. Immunohistochemical study of alpha 1-5 chains of type IV collagen in hereditary nephritis. Kidney international 46, 1413-1421 (1994).

28. Tazon-Vega, B. et al. Genetic testing for X-linked Alport syndrome by direct sequencing of COL4A5 cDNA from hair root RNA samples. American journal of kidney diseases: the official journal of the National Kidney Foundation 50, 257.e251-214, https://doi. org/10.1053/j.ajkd.2007.05.004 (2007).

29. Daga, S. et al. Urine-derived podocytes-lineage cells: A promising tool for precision medicine in Alport Syndrome. Human mutation 39, 302-314, https://doi.org/10.1002/humu.23364 (2018).

30. Doma, M. K. \& Parker, R. RNA quality control in eukaryotes. Cell 131, 660-668, https://doi.org/10.1016/j.cell.2007.10.041 (2007).

31. Tournier, I. et al. A large fraction of unclassified variants of the mismatch repair genes MLH1 and MSH2 is associated with splicing defects. Human mutation 29, 1412-1424, https://doi.org/10.1002/humu.20796 (2008).

32. van der Klift, H. M. et al. Splicing analysis for exonic and intronic mismatch repair gene variants associated with Lynch syndrome confirms high concordance between minigene assays and patient RNA analyses. Molecular genetics \& genomic medicine 3, 327-345, https://doi.org/10.1002/mgg3.145 (2015).

33. Desmet, F. O. et al. Human Splicing Finder: an online bioinformatics tool to predict splicing signals. Nucleic acids research 37, e67, https://doi.org/10.1093/nar/gkp215 (2009).

34. Corvelo, A., Hallegger, M., Smith, C. W. \& Eyras, E. Genome-wide association between branch point properties and alternative splicing. PLoS computational biology 6, e1001016, https://doi.org/10.1371/journal.pcbi.1001016 (2010).

\section{Acknowledgements}

We thank Rebecca Porter, PhD, from Edanz Group (www.edanzediting.com/ac) for editing a draft of this manuscript. This study was supported by Grants-in-Aid for Scientific Research (KAKENHI) from the Ministry of Education, Culture, Sports, Science and Technology of Japan (subject ID: 18K15712 to Tomoko Horinouchi, 19K08726 to Kandai Nozu, 16K19642 to Tomohiko Yamamura and 17H04189 to Kazumoto Iijima), and by AMED under Grant Number JP19ek0109231h0003 to Kandai Nozu and Kazumoto Iijima. Kazumoto Iijima has received grant support from Daiichi Sankyo Co., Ltd., and consulting fees from Takeda Pharmaceutical Company, Ono Pharmaceutical Co., Ltd., Boehringer Ingelheim, Astellas Pharma Inc. and Kyowa Hakko Kirin Co., Ltd. Kandai Nozu has received lecture fees from Novartis Pharmaceuticals Corporation and consulting fees from Kyowa Hakko Kirin Co., Ltd. Kazumoto Iijima and Kandai Nozu have filed a patent application on the development of antisense nucleotides for exon skipping therapy in Alport syndrome.

\section{Author Contributions}

T.H. and T.Y. designed the study concept and wrote the manuscript. Ka.N. interpreted the data and wrote the manuscript. S.M., C.N., N.S., N.M., S.I., Y.A. and R.R. established and conducted molecular analysis and interpreted the data. M.M. established the minigene assay. Ko.N., Y.S., H.N., H.T. and K.I. critically reviewed the manuscript. All authors read and approved the final version of the manuscript. 


\section{Additional Information}

Supplementary information accompanies this paper at https://doi.org/10.1038/s41598-019-48990-9.

Competing Interests: The authors declare no competing interests.

Publisher's note: Springer Nature remains neutral with regard to jurisdictional claims in published maps and institutional affiliations.

(c) (i) Open Access This article is licensed under a Creative Commons Attribution 4.0 International License, which permits use, sharing, adaptation, distribution and reproduction in any medium or format, as long as you give appropriate credit to the original author(s) and the source, provide a link to the Creative Commons license, and indicate if changes were made. The images or other third party material in this article are included in the article's Creative Commons license, unless indicated otherwise in a credit line to the material. If material is not included in the article's Creative Commons license and your intended use is not permitted by statutory regulation or exceeds the permitted use, you will need to obtain permission directly from the copyright holder. To view a copy of this license, visit http://creativecommons.org/licenses/by/4.0/.

(C) The Author(s) 2019 